\title{
Aberration-aware Robust Mask Design with Level-set-based Inverse Lithography
}

\author{
Yijiang Shen, Ngai Wong and Edmund Y. Lam \\ Department of Electrical and Electronic Engineering, \\ The University of Hong Kong, Pokfulam Road, Hong Kong
}

\begin{abstract}
Optical proximity correction (OPC) is one of the most widely used Resolution Enhancement Techniques (RET) in mask designs. Conventional OPC is often designed for a set of nominal imaging parameters without giving sufficient attention to the process variations caused by aspherical wavefront leaving the exit pupil of the lithography system. As a result, the mask designed may deliver poor performance with process variations. In this paper, we first describe how a general point spread function (PSF) with wave aberration can degrade the output pattern quality, and then show how the wave aberration function can be incorporated into an inverse imaging framework for robust input mask pattern design against aberrations. A level-set-based time-dependent model can then be applied to solve it with appropriate finite difference schemes. The optimal mask gives more robust performance against either one specific type of aberration or a combination of different types of aberrations.
\end{abstract}

Keywords: inverse lithography, wave aberration, level-set, Zernike polynomials

\section{INTRODUCTION}

Optical microlithography represents an essential technology for manufacturing Integrated Circuits (IC). Demand for increased density of features on dies and wafers has resulted in the design of circuits with decreasing minimum dimensions. Due to the wave nature of light, as dimensions approach sizes comparable to or smaller than the wavelength of the light used in photolithography process, the bandlimited imaging system introduces undesired distortions and artifacts. Techniques such as Optical Proximity Correction (OPC) attempt to solve this problem by appropriate pre-distortion of the photomask pattern. OPC has been carried out mainly using two approaches: rule-based, or model-based. ${ }^{1}$ In the former, empirical rules are developed to counteract the common problems around pattern corners, edges and local interactions. ${ }^{2,3}$ Rule-based OPC techniques are easy to implement with relatively low cost and high throughput, however, such approaches do not consider the full spectrum of possible photomask patterns, and therefore result in sub-optimal designs. Inverse model-based techniques invert the mathematical model and attempt to directly synthesize the optimized mask pattern, and is not constrained by the topology of the original design..$^{4,5}$ Liu and Zakhor applied branch and bound algorithm ${ }^{6}$ and "bacteria" method ${ }^{7}$ for an optimal binary and phase shifting mask (PSM) design, yet these methods are very timeconsuming. Poonawala and Milanfar designed a model-based OPC system and developed a faster optimization algorithm using steepest-descent ${ }^{8,9}$ which has been further improved by Chan et al. in using an active set method and conjugate gradient, ${ }^{10,11}$ and by Jia et al. in improving the robustness of the design. ${ }^{12,13}$ Meanwhile, level-set method ${ }^{14}$ offers a feasible alternative for inverse lithography. ${ }^{4,15}$ A detailed level-set formulation for inverse lithography ${ }^{16}$ describes the inverse problem as a constrained time-dependent model which is solved using appropriate difference schemes.

While the above-mentioned algorithms enrich the weaponry for solving inverse lithography problems, the current performance still falls short, making this a "virtual virtuality" as it is not yet fully applicable to realworld manufacturing. ${ }^{17,18}$ One such concern is that they are mostly applied to calculate solutions under nominal conditions, without taking process variation into account. With a lower $k_{1}$ factor, process variations have caused

Further author information:

Yijiang Shen: E-mail: yjshen@eee.hku.hk, Telephone: 852 2859-2696

Ngai Wong: E-mail: nwong@eee.hku.hk, Telephone: 852 2859-1914

Edmund Y. Lam: E-mail: elam@eee.hku.hk, Telephone: 852 2241-5942

Photomask and Next-Generation Lithography Mask Technology XVII, edited by Kunihiro Hosono,

Proc. of SPIE Vol. 7748, 77481U · @ 2010 SPIE · CCC code: 0277-786X/10/\$18 · doi: 10.1117/12.863973

Proc. of SPIE Vol. 7748 77481U-1 
more critical dimensions (CD) variations and hotspots, which deserve more attention. ${ }^{18}$ Consequently, various efforts have been made to incorporate process conditions into OPC algorithms for robust design of printed patterns. ${ }^{12,13,19}$ Another major source of CD variation other than defocus and dose variation is aberration ${ }^{20}$ which is defined as deviation of wavefronts from sphericity. The aberration function can be expressed in terms of a complete set of polynomials, namely, Zernike polynomials. ${ }^{21,22}$ However, the applicability of the original Nijboer-Zernike theory is limited mainly to wave-front deviations well below the value of one wavelength. An extended Nijboer-Zernike approach ${ }^{23,24}$ is proposed to yield point-spread function of the optical systems in the case of large aberrations and defocus values.

In this paper, we propose a statistical method which incorporates process variations including both defocus and aberrations into the inverse lithography problem. The method treats defocus and aberrations as random variables, taking their statistical properties into account, and develops an optimization set which aims to minimize the pattern fidelity difference between the printed wafer on the pattern upon various defocus and aberration conditions and the target pattern. A complete level-set formulation of the optimization problem is provided with a computationally efficient solution to the proposed level-set-based formulation. ${ }^{16}$

This paper is organized as follows. Section 2 describes the mathematical model which incorporates defocus and aberrations into the inverse lithography problem. Section 3 introduces level-set-based optimization framework. Experimental results are presented in Section 4. Finally, Section 5 draws concluding remarks and provides some future insights.

\section{MATHEMATICAL MODEL}

For simplicity in both mathematics and implementation, we describes the problem formation using coherent sources and binary masks, but our methodology can be readily applied to partially coherent illumination and PSMs. The projection lithography imaging process can be described as a forward model

$$
I(\mathbf{x})=\mathcal{T}\{U(\mathbf{x})\},
$$

where the boldface $\mathbf{x}$ denotes spatial coordinates $(x, y)$, and $\mathcal{T}\{\cdot\}$ maps the input intensity function $U(\mathbf{x})$ to the output intensity function $I(\mathbf{x})$. Due to the lowpass nature of the optical lithography imaging system, $I(\mathbf{x})$ is typically a blurred version of $U(\mathbf{x})$. Suppose we denote the desired circuit pattern as $I_{0}(\mathbf{x})$. The objective of inverse lithography is to find a predistorted input intensity function $\hat{U}(\mathbf{x})$ which minimizes its distance with the desired output, i.e.,

$$
\hat{U}(\mathbf{x})=\underset{U(\mathbf{x})}{\arg \min } d\left(I_{0}(\mathbf{x}), \mathcal{T}\{U(\mathbf{x})\}\right),
$$

in which $d(\cdot, \cdot)$ is an appropriately defined distance metric, such as the $\ell_{2}$ norm.

The lithography process $\mathcal{T}\{\cdot\}$ in Eq. (1) can be divided into two functional blocks, namely the projection optics effects (aerial image formation) and resist effects. If the optical system is coherent, the aerial image intensity distribution $I_{\text {aerial }}(\mathbf{x})$ is related to the input intensity function $U(\mathbf{x})$ and the point spread function (PSF) $H(\mathbf{x})$ by

$$
I_{\text {aerial }}(\mathbf{x})=|H(\mathbf{x}) * U(\mathbf{x})|^{2},
$$

where $*$ denotes convolution, and $H(\mathbf{x})$ in an ideal case where there exist no defocus and aberrations, is taken as the inverse Fourier transform of a disc function. ${ }^{25}$ The resist effects can be approximated using a logarithmic sigmoid function ${ }^{9}$

$$
\operatorname{sig}(U(\mathbf{x}))=\frac{1}{1+\mathrm{e}^{-a\left(U(\mathbf{x})-t_{r}\right)}},
$$

with $a$ being the steepness of the sigmoid and $t_{r}$ being the threshold. Putting together, we can write the image formation equation for a coherent imaging system as

$$
I(\mathbf{x})=\mathcal{T}\{U(\mathbf{x})\}=\operatorname{sig}\left(I_{\text {aerial }}(\mathbf{x})\right)=\operatorname{sig}\left(|H(\mathbf{x}) * U(\mathbf{x})|^{2}\right) .
$$




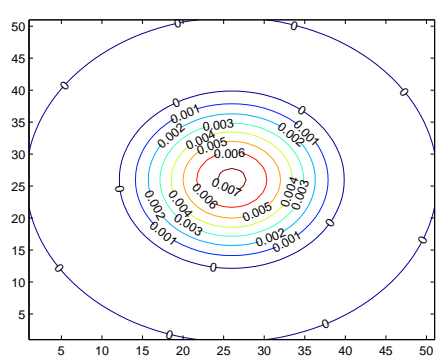

(a)

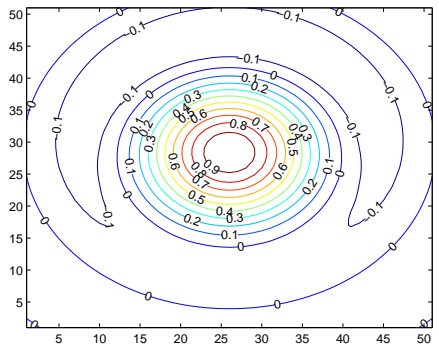

(b)

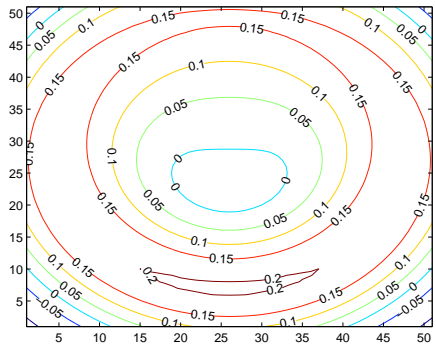

(c)

Figure 1. PSF with (a) no defocus and no aberration, (b) no defocus but coma aberration with $c_{31}=10$ and (c) defocus $f=20 \pi$ and coma aberration with $c_{31}=10$.

In what follows, we will drop the arguments $\mathbf{x}$ whenever there is no ambiguity. In the normalized form that is convenient for our purposes, the diffraction integrals that we are concerned with $H$ take the form

$$
H=\frac{1}{\pi} \int_{0}^{1} \int_{0}^{2 \pi} \mathrm{e}^{2 \pi j \rho r \cos (\theta-\phi)} \mathrm{d} \theta \mathrm{d} \rho
$$

where $j=\sqrt{-1}, \rho, \theta$ and $r, \phi$ are the polar coordinates in the exit pupil function and in the image plane, respectively. $H$ is characterized by the function

$$
H(r)=2 \frac{J_{1}(2 \pi r)}{2 \pi r},
$$

also known as the Airy pattern, where $J_{1}$ is the Bessel function of the first kind. However, when defocus and aberrations are present, the diffraction pattern concerning $H$ becomes

$$
H=\frac{1}{\pi} \int_{0}^{1} \rho \mathrm{e}^{j f \rho^{2}} \times\left\{\int_{0}^{2 \pi} \mathrm{e}^{j \Phi(\rho, \theta+\phi)} \times \mathrm{e}^{2 \pi j \rho r \cos \theta} \mathrm{d} \theta\right\} \mathrm{d} \rho,
$$

with $f$ being defocus, and $\Phi$ being the aberration function which can be expanded using Zernike polynomials according to the Nijboer-Zernike theory as

$$
\Phi(\rho, \theta)=\sum_{n, m} c_{n m} R_{n}^{m}(\rho) \cos m \theta,
$$

in which $c_{n m} R_{n}^{m}(\rho) \cos m \theta$ is a polynomial of the Zernike set. The first fifteen polynomials of the Zernike set can be found in Ref 25 .

According to Ref 23 and 24, the diffraction pattern $H$ in Eq. (8) can be expressed in terms of

$$
T_{n m}:=\int_{0}^{1} \rho^{n+1} \mathrm{e}^{j f \rho^{2}} J_{m}(2 \pi r \rho) \cos m \phi \mathrm{d} \rho .
$$

Thus, the integral expression for $H$ in Eq. (8) can be expressed explicitly, in terms of powers of $f, c_{n m}$ in Eq. (9), and Bessel functions, in a way that is, in principle, practical for computerization. In Fig. 1, the contour plots of the PSF with (a) no defocus and no aberration, (b) no defocus but coma aberration with $c_{31}=1$ and (c) defocus $f=20 \pi$ and coma aberration with $c_{31}=10$ are presented as examples to show how defocus and aberration can effect the PSF under nominal condition.

\section{LEVEL-SET-BASED OPTIMIZATION FRAMEWORK}

In optical lithography, the image does not always lie on the focal plane, but may vary randomly around it. In other words, the defocus $f$ between the image plane and focal plane behaves like a random variable. In the 
Nijboer-Zernike theory, defocus can also be represented as Zernike polynomials. ${ }^{25}$ As a reasonable assumption, the coefficients are modeled as independent, normally distributed random variables with zero mean and identical non-zero variance. The root-mean-square (rms) deformation

$$
\Delta \Phi_{r m s}=\sqrt{\sum_{n, m} c_{n m}^{2}},
$$

becomes a $\chi^{2}$ (chi-square distribution) random variable with degree of freedom equal to the number of Zernike polynomials in the combined aberration.

The statistical method proposed in this paper states that in order to optimize the average performance of layouts, the objective function takes expectations of the difference under various aberration conditions with a certain distribution of the rms deformation. The optimization problem we aim to solve becomes

$$
U_{\text {optimal }}=\min _{U} \mathcal{E}\left\{\left\|I-I_{0}\right\|_{2}^{2}\right\},
$$

where $\|\cdot\|_{2}^{2}$ denotes $\ell_{2}$ norm and $\mathcal{E}$ denotes expectation. Various algorithms have been developed to solve the inverse lithography problem in Eq. (12), including steepest descent and conjugate gradient. ${ }^{26}$ Alternatively, a level-set-based inverse lithography technology (ILT) has been developed to solve the ILT problem, ${ }^{16}$ the advantage of which being pattern error improvement and elimination of unwanted small blocks in mask manufacturing. As such, a stable explicit time-dependent model is applied,

$$
\frac{\partial U}{\partial t}=-|\nabla U| \alpha(\mathbf{x}, t),
$$

in which $\alpha(\mathbf{x}, t)$ is computed as

$$
\begin{aligned}
\alpha(\mathbf{x}) & =\frac{1}{2} \frac{\partial}{\partial U}\left(\operatorname{sig}\left(|H * U|^{2}\right)-I_{0}\right)^{2} \\
& =a \times \mathcal{E}\left\{H *\left[\left(I_{0}-\operatorname{sig}\left(|H * U|^{2}\right)\right) \odot \operatorname{sig}\left(|H * U|^{2}\right) \odot\left(1-\operatorname{sig}\left(|H * U|^{2}\right)\right) \odot(H * U)\right]\right\},
\end{aligned}
$$

where $\odot$ denotes element-by-element multiplication. One can immediately notice that Eq. (13) is a partial differential equation (PDE) which could be solved by various finite difference schemes. The authors suggest that first-order temporal accuracy and second-order essentially nonoscillatory spatial accuracy are appropriate difference schemes to compute the PDE. ${ }^{16}$ The computation stops after a certain number of iterations or when the value of the cost function has decreased below a certain threshold value.

It should be noted that by employing the expectation operator, the average amount of change under different defocus and aberration conditions is incorporated into the time-dependent model in Eq. (13). Therefore, it optimizes the mask pattern over the defocus range and aberration coefficients, which makes it more robust against defocus and aberrations than an optimization at nominal conditions.

\section{EXPERIMENTAL RESULTS}

We apply the proposed level-set-based aberration-aware ILT to design binary masks which are robust against different aberrations. System parameters are set as follows: $\lambda=193 \mathrm{~nm}, \mathrm{NA}=0.85$, resolution $=10 \mathrm{~nm} /$ pixel, PSF $H$ is a jinc function of the same size as the required pattern, steepness of the sigmoid function $a=85$, thresholding $t_{r}=0.3$. The algorithm stops after 400 iterations.

In one set of photomask synthesis simulations, the proposed algorithm is applied to intended target pattern in Fig. 2(a) for aberration-aware input mask pattern against coma. The Zernike coefficient associated with this coma aberration is $c_{31}$, which when it is the only aberration present without defocus, is defined as a normally distributed variable with zero mean and nonzero variance. Fig. 2(b) shows the coma-degraded output pattern using the target pattern in Fig. 2(a), suggesting an off-axis effect on output pattern when $c_{31}=100$. Fig. 2(c) shows the optimal input mask pattern computed under nominal condition, i.e., when there is no aberration 


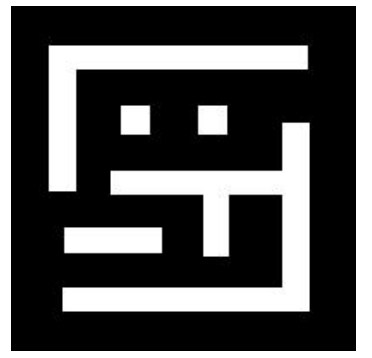

(a)

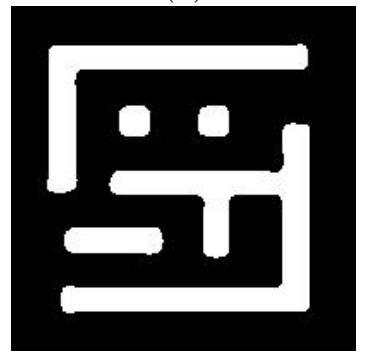

(e)

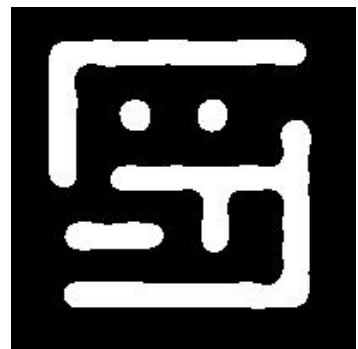

(b)

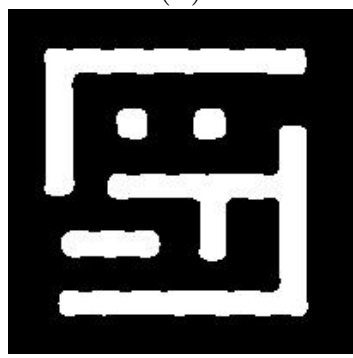

(f)

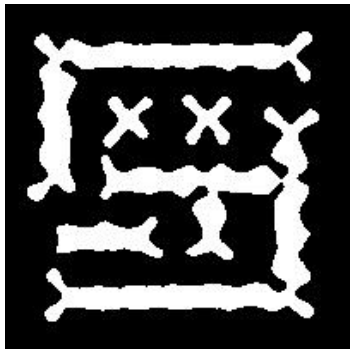

(c)

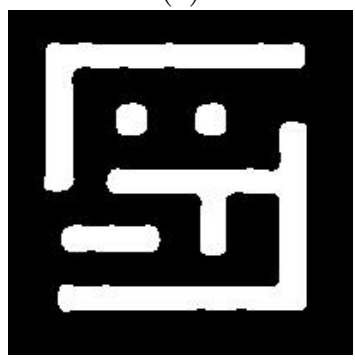

$(\mathrm{g})$

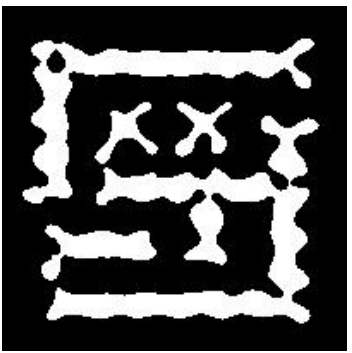

(d)

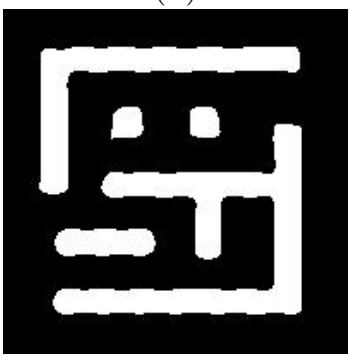

(h)

Figure 2. Simulation with optimal input mask under nominal condition and optimal input mask against coma. (a) Target pattern. (b) Output mask under coma $\left(c_{31}=100\right)$ with input (a). (c) Optimal input mask under nominal condition. (d) Optimal input mask against coma. (e) Output mask under nominal condition with input (b). (f) Output mask under nominal condition with input (c). (g) Output mask under coma $\left(c_{31}=100\right)$ with input (b). (h) Output mask under coma $\left(c_{31}=100\right)$ with input (c).

Table 1. Pattern error in Fig. 2

\begin{tabular}{cccccc}
\hline \hline Experiments & Fig. 2(b) & Fig. 2(e) & Fig. 2(f) & Fig. 2(g) & Fig. 2(h) \\
\hline Pattern Error & 3275 & 231 & 416 & 3030 & 725 \\
\hline \hline
\end{tabular}




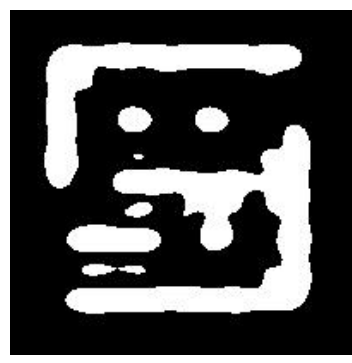

(a)

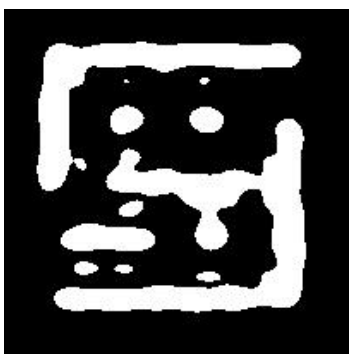

(b)

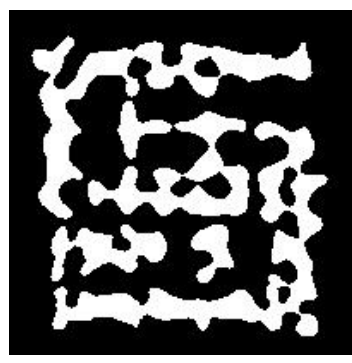

(c)

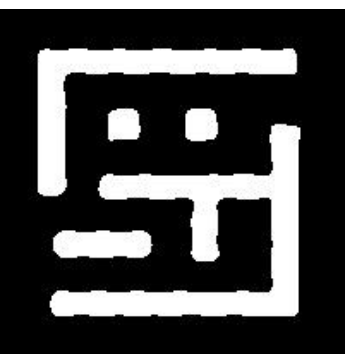

(d)

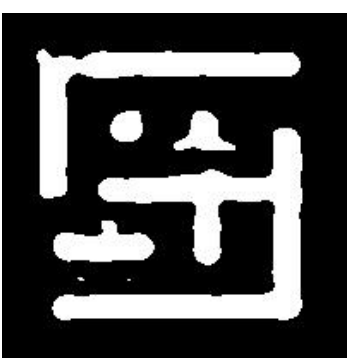

(e)

Figure 3. Simulation with optimal input mask under nominal condition and optimal input mask against defocus and coma. (a) and (b) Coma-defocus-degraded output pattern with input Fig. 2(a)and Fig. 3(b) under defocus $f=800$ and coma $c_{31}=80$, respectively. (c) Optimal input mask against defocus and coma. (d) Output mask under nominal condition with input (c). (e) Output mask under coma defocus $f=800$ and coma $c_{31}=80$ with input (c).

Table 2. Pattern error in Fig. 3

\begin{tabular}{ccccc}
\hline \hline Experiments & Fig. 3(b) & Fig. 3(c) & Fig. 3(f) & Fig. 3(g) \\
\hline Pattern Error & 3752 & 3669 & 797 & 884 \\
\hline \hline
\end{tabular}

at all. Fig. 2(d) shows the aberration-aware input mask pattern out of the proposed algorithm. Fig. 2(e) and (f) are the resulting output pattern under nominal condition with Fig. 2(b) and (c) as input respectively. Fig. $2(\mathrm{~g})$ and $(\mathrm{h})$ are the resulting output pattern under coma when $c_{31}=100$. Pattern error of simulation results in Fig. 2 is given in Table 1, where pattern error is examined numerically by counting how many pixels are different between the intended circuit pattern and the one obtained after the simulated lithographic system. From Fig. 2 and Table 1, it is concluded that while under nominal conditions, the optimal input mask pattern under nominal condition outperforms the proposed aberration-aware input mask, which is not a surprise since the former is intended specifically for nominal conditions and the latter is not, the proposed aberration-aware input mask greatly improves pattern fidelity against coma, with much less pattern error than the optimal input mask pattern computed under nominal condition. These observations justify the proposed algorithm in producing aberration-aware input mask pattern for critical structures.

Another set of simulation is given in Fig. 3 to compute aberration-aware input mask against defocus and coma. Fig. 3(a) and (b) show the coma-defocus-degraded output patterns using target pattern in Fig. 2(a), and optimal input mask pattern computed under nominal condition in Fig. 2(c) as input pattern under defocus $f=800$ and coma $c_{31}=80$, respectively. Fig. 3(c) shows the computed aberration-aware mask. Fig. 3(d) and (e) are the resulting output pattern with Fig. 3(c) as input under nominal conditions and under defocus $f=800$ and coma $c_{31}=80$, respectively. Pattern error of the simulation results in Fig. 3(c) is given in Table 2 . Observations from this set of simulation show that the proposed algorithm produces input masks which improve pattern fidelity against coma and defocus.

It should be noted that in the above-mentioned simulations, the defocus parameter $f$ and aberration coefficients $c_{n m}$ are chosen to be relatively large. However, when the aberrations are modest, the aberration-aware 
present PSF can be computed using an approximation to enhance processing speed. ${ }^{23,24}$ Relatively large aberrations are used to better show the robustness against aberrations of the proposed method. It should also be noted that when more aberrations other than coma are present, the root mean square deformation of the combined aberration becomes a chi-square distribution, and the proposed algorithm will produce aberration-aware input masks against this combined aberration similarly.

\section{CONCLUSION}

In this paper, we propose a level-set-based ILT algorithm incorporating statistical variations of different aberrations into the inverse imaging system. Point spread function with aberrations is computed explicitly, and the inverse problem is solved efficiently using level-set-based time-dependent model. Experimental results show that the proposed method produce aberration-aware input mask pattern which shows great robustness against aberrations.

\section{ACKNOWLEDGMENTS}

This work was supported in part by the Research Grants Council of the Hong Kong Special Administrative Region, China under Projects HKU 7139/06E, 7174/07E, 7134/08E and 7185/09E.

\section{REFERENCES}

[1] A. K.-K. Wong, Resolution Enhancement Techniques in Optical Lithography, SPIE Press, Bellingham, WA, 2001.

[2] O. W. Otto, J. G. Garofalo, K. K. Low, C.-M. Yuan, R. C. Henderson, C. Pierrat, R. L. Kostelak, S. Vaidya, and P. K. Vasudev, "Automated optical proximity correction: a rules-based approach," in Proc. SPIE, 2197, pp. 278-293, 1994.

[3] S. Shioiri and H. Tanabe, "Fast optical proximity correction: analytical method," in Proc. SPIE, 2440, pp. 261-269, 1995.

[4] L. Pang, Y. Liu, and D. Abrams, "Inverse lithography technology (ILT): a natural solution for model-based SRAF at 45nm and 32nm," in Proc. SPIE, 6607, p. 660739, 2007.

[5] S. H. Chan, A. K. Wong, and E. Y. Lam, "Initialization for robust inverse synthesis of phase-shifting masks in optical projection lithography," Opt. Express 16(19), pp. 14746-14760, 2008.

[6] Y. Liu and A. Zakhor, "Optimal binary image design for optical lithography," in Proc. SPIE, 1264, pp. 401$412,1990$.

[7] Y. Liu and A. Zakhor, "Binary and phase-shifting image design for optical lithography," in Proc. SPIE, 1463, pp. 382-399, 1991.

[8] A. Poonawala and P. Milanfar, "Prewarping techniques in imaging: applications in nanotechnology and biotechnology," in Proc. SPIE, 5674, pp. 114-127, 2005.

[9] A. Poonawala and P. Milanfar, "Mask design for optical microlithography: an inverse imaging problem," IEEE Trans. Image Process. 16(3), pp. 774-788, 2007.

[10] S. H. Chan and E. Y. Lam, "Inverse image problem of designing phase shifting masks in optical lithography," in Proceedings of IEEE International Conference on Image Processing, pp. 1832-1835, 2008.

[11] S. H. Chan, A. K. Wong, and E. Y. Lam, "Inverse synthesis of phase-shifting mask for optical lithography," in OSA Topical Meeting in Signal Recovery and Synthesis, p. SMD3, 2007.

[12] N. Jia, A. K. Wong, and E. Y. Lam, "Robust photomask design with defocus variation using inverse synthesis," in Proc. SPIE, 7140, p. 71401W, 2008.

[13] N. Jia, A. K. Wong, and E. Y. Lam, "Regularization of inverse photomask synthesis to enhance manufacturability," in Proc. SPIE, 7520, p. 752032, 2009.

[14] S. Osher and R. P. Fedkiw, "Level set methods: an overview and some recent results," J. Comput. Phys. 169(2), pp. 463-502, 2001.

[15] L. Pang, G. Dai, T. Cecil, T. Dam, Y. Cui, P. Hu, D. Chen, K. Baik, and D. Peng, "Validation of inverse lithography technology (ILT) and its adaptive SRAF at advanced technology nodes," in Proc. SPIE, 6924, p. $69240 \mathrm{~T}, 2008$. 
[16] Y. Shen, N. Wong, and E. Y. Lam, "Level-set-based inverse lithography for photomask synthesis," Opt. Express 17(26), pp. 23690-23701, 2009.

[17] E. Y. Lam and A. K. Wong, "Computation lithography: virtual reality and virtual virtuality," Opt. Express 17(15), pp. 12259-12268, 2009.

[18] A. K. Wong and E. Y. Lam, "The nebulous hotspot and algorithm variability," in Proc. SPIE, 7275, p. $727509,2009$.

[19] N. Jia and E. Y. Lam, "Machine learning for inverse lithography: Using stochastic gradient descent for robust photomask synthesis," J. Opt. in press , 2010.

[20] A. Wong, A. Molless, T. Brunner, E. Coker, R. Fair, G. Mack, and S. Mansfield, "Characterization of linewidth variation," in Proc. SPIE, 4000, p. 184, 2000.

[21] B. Nijboer, The diffraction theory of aberrations. PhD thesis, Groningen University, 1942.

[22] R. J. Noll, "Zernike polynomials and atmospheric turbulence," J. Opt. Soc. Am. A 66(3), pp. 207-211, 1976.

[23] J. Braat, P. Dirksen, and A. Janssen, "Assessment of an extended Nijboer-Zernike approach for the computation of optical point-spread functions," J. Opt. Soc. Am. A 19(5), pp. 858-870, 2002.

[24] P. Dirksen, J. Braat, A. Janssen, and C. Juffermans, "Aberration retrieval using the extended NijboerZernike approach," J. Microlith. Microfab. Microsys. 2, p. 61, 2003.

[25] A. K.-K. Wong, Optical Imaging in Projection Microlithography, SPIE Press, Bellingham, WA, 2005.

[26] M. Minoux, Mathematical Programming: Theory and Algorithms, Wiley, New York, 1986. 